\title{
Proyecto para la construcción de un precalentador de lecho fluidizado para un horno piloto de laboratorio
}

ANGEL PALOMO SANCHEZ Ledo. en Ciencias Quimicas (IETCC/CSIC)

\section{$R E S U M E N$}

Se provecta un precalentador en lecho fluidizado a escala piloto, capaz de acoplarse a un horno rotatorio, también piloto, para la fabricación de clinker portland.

Con ello se pretende el diseño de un sistema de simulación dinámica de clinkerización, para conseguir clinker de caracteristicas similares a las que se obtienen industrialmente.
$S U M M A R Y$

A preheater with fluidized bed is planned on a pilot scale, capable of being fitted to a rotatory furnace, also of a pilot nature, for the manufacture of portland clinker.

With this it is intended to design a system of dynamic simulation of clinkerization, to obtain clinker of similar characteristics to those which are obtained industrially.

\section{IN T R O D U C C I O N}

El proceso de fabricación de los conglomerantes hidráulicos consta de un tratamiento térmico fuerte de las materias primas y posteriormente de una molienda fina de los materiales resultantes de la cocción con o sin otras adiciones.

Este tratamiento térmico que sufren las materias primas se conoce con el nombre de "Clinkerización". En la actualidad este proceso de clinkerización es un tema puntal en la investigación que sobre el cemento se realiza en el mundo; entre otras razones por las importantes implicaciones económicas que acarrea en materia energética.

En la industria, la clinkerización se lleva a cabo en unos hornos que pueden ser de dos tipos: vertical y rotatorio. El rotatorio es el más utilizado actualmente. Este tipo de horno fue introducido por Frederik Ronsome en 1885 y en sus origenes estaba calentado por gas (entonces no se conocia la calefacción por carbón) y tenía unas dimensiones de 1,8-2 $\mathrm{m}$ de diámetro por 20-25 m de longitud con caudales de 30-50 t/dia.

Parece lógico pensar que la evolución y creación de la técnica asociada a los conglomerantes hidráulicos, así como el conocimiento de éstos en su constitución y propiedades, hayan mantenido una estrecha relación con el desarrollo de la Ciencia y de la Tecnologia a través de los tiempos. Precisamente, de este contacto investigación-desarrollo es de donde mana el objeto de este Proyecto.

Como anteriormente se apuntaba, se ha hecho imprescindible conocer en profundidad el proceso de fabricación del cemento Portland por el cual, las materias primas mezcladas, molidas y homogeneizadas sufren un tratamiento térmico que los convierte en el clinker de cemento Portland. 
A pesar de que hoy en día son muchos los centros de investigación extendidos por el mundo que de una forma u otra están relacionados con el cemento como material a investigar y a mejorar, la traslación de determinados problemas desde el laboratorio de investigación a la industria y viceversa resulta inviable por la falta de semejanza del instrumental empleado en uno y otro sitio. Es el caso de los hornos. Existen en el mercado gran cantidad de modelos de hornos de laboratorio que presentan los últimos avances tecnológicos y que en definitiva facilitan y mejoran la labor del investigador. Sin embargo, todos ellos adolecen de una propiedad fundamental que tienen los hornos de las fábricas de cemento: el movimiento.

No es la primera vez que se plantea el problema de pasar por una etapa intermedia entre el laboratorio y la fábrica, pero la experiencia indica que los hornos de planta piloto (aquellos de los que se conoce su existencia) no han llegado a dar el rendimiento adecuado.

\section{AN TECEDENTES}

Existen diversos procedimientos industriales para someter las materias primas (en general caliza y arcilla convenientemente molidas, homogeneizadas y dosificadas) al necesario tratamiento térmico para que se lleven a cabo las reacciones que conducirán a la obtención del clinker.

Están ampliamente extendidos sistemas que tienen por objeto realizar una precalcinación del crudo antes de su incorporación al horno rotatorio. De gran importancia es la técnica del intercambiador de calor por suspensión de los crudos en gases. Se alcanzan temperaturas próximas a los $900^{\circ} \mathrm{C}$, con un considerable aprovechamiento energético.

\section{La fluidización en la calcinación de crudos de cemento}

La fluidización es la operación por la cual unos sólidos adquieren un estado fluido gracias al contacto de dichos sólidos con un gas o un líquido.

Las caracteristicas poco usuales del contacto sólido-fluido hacen que la ingeniería de la fluidización haya adquirido una gran importancia en numerosos procesos industriales (Gasificacion del carbón, Reducción del mineral de hierro, Producción de cemento, etc.). Estas características, se pueden resumir en las siguientes ventajas e inconvenientes:

\section{VENTAJAS}

- Facilidad de alcanzar condiciones próximas a las isotermas.

- Adecuación a operaciones de gran escala.

- Alta velocidad de transferencia de calor y materia entre el gas y las partículas.

\section{INCONVENIENTES}

- Rápida mezcla de sólidos en el lecho que conduce a tiempos de residencia no uniformes.

- Los sólidos poco compactos se pulverizan y son arrastrados por el gas.

- Las partículas pueden erosionar seriamente las paredes del conducto.

En cualquier caso las ventajas superan notablemente a los inconvenientes, de modo que la calcinación de crudos de cemento en lechos fluidizados podria quedar justificada como sigue:

La producción de cemento en el mundo tiene una velocidad de crecimiento aproximadamente 
igual al crecimiento de la población mundial, lo que significa que cada semana en algún lugar del mundo entra en funcionamiento una nueva fábrica de cemento con una capacidad de producción de 500.000 t/año. Esto supone un gasto de energía tal, que el desarrollo y puesta en práctica de procesos de calcinación lo más eficientes posibles quedan sobradamente justificados.

En los años que siguieron al término de la 2. ${ }^{a}$ guerra mundial, la calcinación era una etapa más dentro del conjunto de etapas que comprendia la producción de cemento (secado, precalentamiento, calcinación, clinkerización y enfriamiento) y que se desarrollaban con mayor o menor efectividad dentro del mismo "reactor": el horno rotatorio.

El desarrollo en los años que siguieron cambió sustancialmente la tecnología y en la actualidad los métodos más modernos que emplean la precalcinación ya han recorrido un largo camino hacia el encuentro de los requisitos necesarios para que los cuatro procesos básicos (precalentamiento, calcinación, clinkerización y enfriamiento) puedan llevarse a cabo en "unidades" de reacción separadas.

Cálculos realizados con temperaturas constantes (para calcinación completa) comprendidas entre 620 y $900^{\circ} \mathrm{C}$ confirmaron el hecho observado de que la calcinación de un crudo es el parámetro que de manera más importante incide en el gasto total de energía dentro de la producción de cemento. Se encontró que era posible sustituir más del $60 \%$ de la energía consumida en una planta por un calcinador; lo que significa que este sistema de calefacción en principio "secundario" se convierte en el sistema principal de calefacción del proceso.

La explicación a tan importante hecho se debe fundamentalmente a 4 fenómenos que se dan en los calcinadores:

- Descomposición completa de carbonato cálcico.

$$
\mathrm{CaCO}_{3} \leftrightarrows \mathrm{CaO}+\mathrm{CO}_{2} \quad H_{0}=-178 \mathrm{KJ} / \mathrm{mol}
$$

- Combustión completa de fuels con alto contenido en cenizas.

- Muy buen control de la temperatura.

- Retención de sólidos suficientemente grande en el tiempo.

Además, los calcinadores se caracterizan por crear un bajo nivel de contaminación.

\section{DISEÑO DE UN LECHO FLUIDIZADO}

\section{Velocidad Mínima de fluidización}

Supongamos un fluido que pasa a través de un lecho de finas partículas. Cuando la velocidad del fluido es baja, éste se limita a filtrarse por los espacios o huecos que hay entre las partículas. Si se aumenta un poco la velocidad del fluido, las partículas comienzan a vibrar y a moverse en regiones limitadas. A velocidades todavía mayores, se alcanza un punto en el que las partículas quedan suspendidas sobre el fluido. En este punto, la fuerza de arrastre debida al movimiento ascendente del fluido es igual al peso de las partículas; la componente vertical de la fuerza de compresión entre partículas adyacentes desaparece y la caida de presión en cualquier sección del lecho es igual al peso del fluido y de las partículas en esa sección. En este punto se dice que el lecho está fluidizado a la mimima velocidad de fluidización.

Matemáticamente, las condiciones de mínima fluidización se pueden expresar como:

$$
A P / L_{\mathrm{mf}}=\left(1-\varepsilon_{\mathrm{mf}}\right)\left(\rho_{\mathrm{s}}-\rho_{\mathrm{g}}\right) g / g_{\mathrm{c}}
$$


Y de aqui, la velocidad mínima de fluidización para cualquier número de Reynolds:

$$
\frac{d_{\mathrm{p}} \mu_{\mathrm{mr}} \rho_{\mathrm{g}}}{\mu}=\left[(33,7)^{2}+0,0408 \frac{d_{\mathrm{p}}^{3} \rho_{\mathrm{g}}\left(\rho_{\mathrm{s}}-\rho_{\mathrm{g}}\right) g}{\mu^{2}}\right]^{1 / 2}-33,7
$$

en donde:

$d_{\mathrm{p}}=$ tamaño medio de partícula;

$\mu_{\mathrm{mf}}=$ velocidad mínima de fluidización;

$\rho_{\mathrm{g}}=$ densidad del gas;

$\rho_{\mathrm{s}}=$ densidad del sólido;

$\mu=$ viscosidad del gas;

$g \quad=$ aceleración de la gravedad.

En el caso que se esta tratando, todos estos datos serán conocidos:

- El tamaño medio de partícula $\left(d_{\mathrm{p}}\right)$ se calculó experimentalmente a partir de un crudo de cemento portland que se puede considerar típico. $100 \mathrm{~g}$ de este crudo fueron tamizados obteniéndose los siguientes resultados:

\begin{tabular}{|c|c|}
\hline Abertura de malla del tamiz en micras & Cantidad de crudo retenida en el tamiz en $\mathbf{~}$ \\
\hline 297 & 0,3400 \\
210 & 1,1010 \\
149 & 4,2437 \\
105 & 7,7549 \\
74 & 10,2940 \\
62 & 5,9414 \\
37 & 24,0626 \\
Fondo & 7,1630 \\
\hline
\end{tabular}

De aquí se construyó la tabla de pesos acumulativos:

\begin{tabular}{|c|c|}
\hline Peso acumulativo (P) & Con diámetro más pequeño que $\mathbf{d}_{\mathbf{p}}$ \\
\hline 38,8994 & 37 \\
46,0624 & 44 \\
70,1250 & 62 \\
76,0664 & 74 \\
86,3604 & 105 \\
94,1153 & 149 \\
98,3590 & 210 \\
99,4600 & 297 \\
\hline
\end{tabular}

La representación de $P$ frente a $d_{\mathrm{p}}$ da la curva de distribución acumulativa (fig. 1) que da idea de la cantidad de partículas en la muestra, con tamaño inferior a uno dado. 


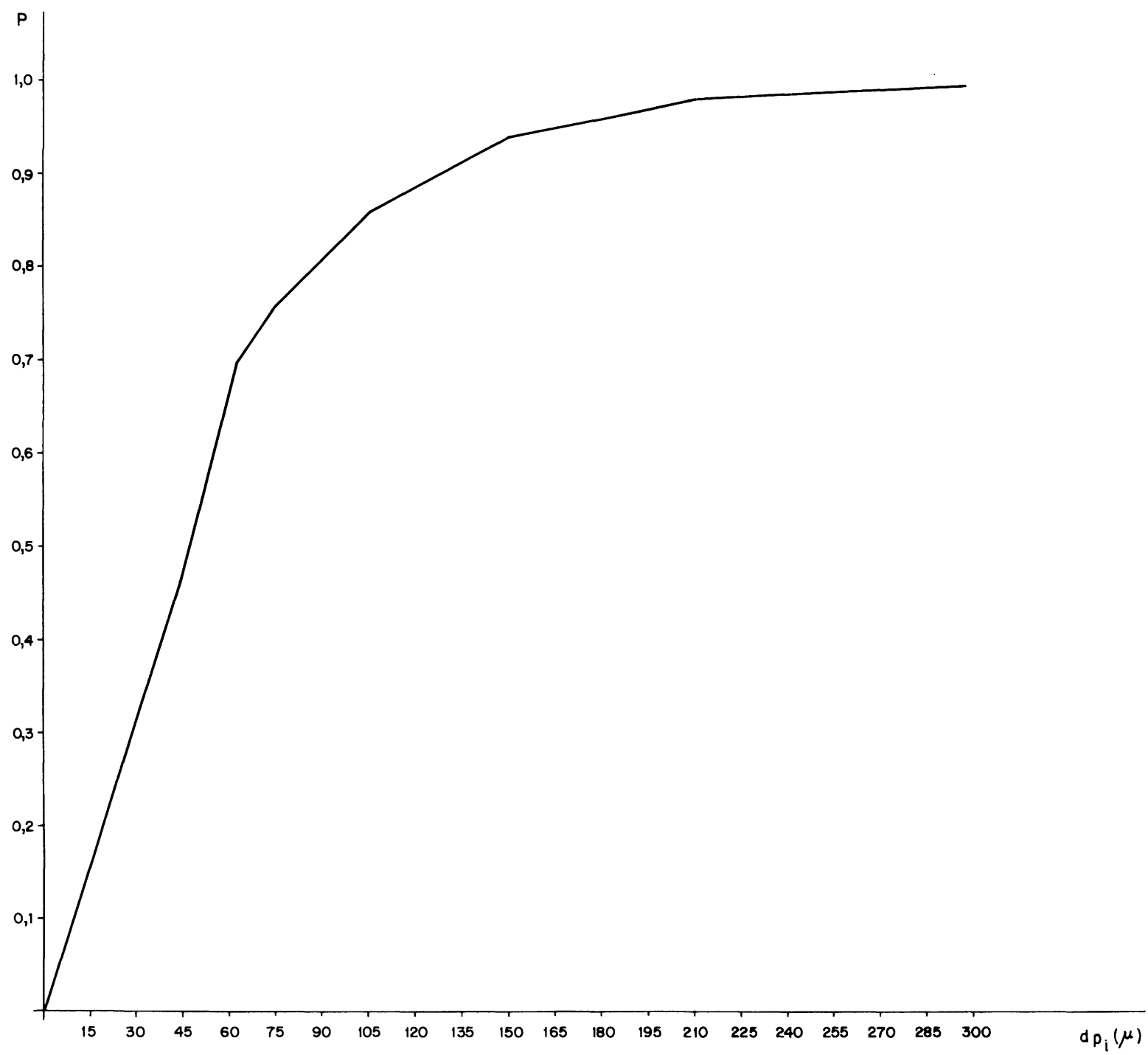

Fig. 1

El tamaño medio de partículas se calcula mediante la fórmula:

$$
\bar{d}_{\mathrm{p}}=\frac{1}{\Sigma\left(X / d_{\mathrm{p}}\right)_{\mathrm{i}}}
$$

Es necesario pues recurrir a la tabla I.

T A B LA I

\begin{tabular}{|c|c|c|cc|}
\hline $\begin{array}{c}\text { Rango de diámetros } \\
(\mu)\end{array}$ & $\begin{array}{c}\mathbf{d}_{\mathbf{p}_{\mathbf{i}}} \\
(\mu)\end{array}$ & $\begin{array}{c}\text { Fracción de peso en el intervalo } \\
\mathbf{X}_{\mathbf{i}}=\left(\mathbf{P} \Delta \mathbf{d}_{\mathbf{p}} \mathbf{~}_{\mathbf{i}}\right.\end{array}$ & $\mathbf{( \mathbf { X } / \mathbf { d } _ { \mathbf { p } } )}$ \\
\hline $37-44$ & 40,5 & $(46,0624-38,8994) / 100=0,07163$ & $0,07163 / 40,5=0,001768$ \\
$44-62$ & 53 & $(70,1250-46,0624) / 100=0,24062$ & $, 0,24062 / 53$ & $=0,004540$ \\
$62-74$ & 68 & $(76,0664-70,1250) / 100=0,05941$ & $0,05941 / 68$ & $=0,000873$ \\
$74-105$ & 89,5 & $(86,36040-76,0664) / 100=0,10294$ & $0,10294 / 89,5=0,001150$ \\
$105-149$ & 127 & $(94,1153-86,3604) / 100=0,07754$ & $0,07754 / 127=0,000610$ \\
$149-210$ & 179,5 & $(98,3590-94,1153) / 100=0,04243$ & $0,04243 / 179,5=0,000236$ \\
$210-297$ & 253,5 & $(99,4600-98,3590) / 100=0,01101$ & $0,01101 / 253,5=0,000043$ \\
& & & & 0,009220 \\
\hline
\end{tabular}


Por tanto:

$$
\bar{d}_{\mathrm{p}}=\frac{1}{0,009220}=108,46 \mu
$$

En la fig. 2 se representa el rango de diámetros frente a la fracción de peso en el intervalo, lo que da idea de la distribución del tamaño de partículas.

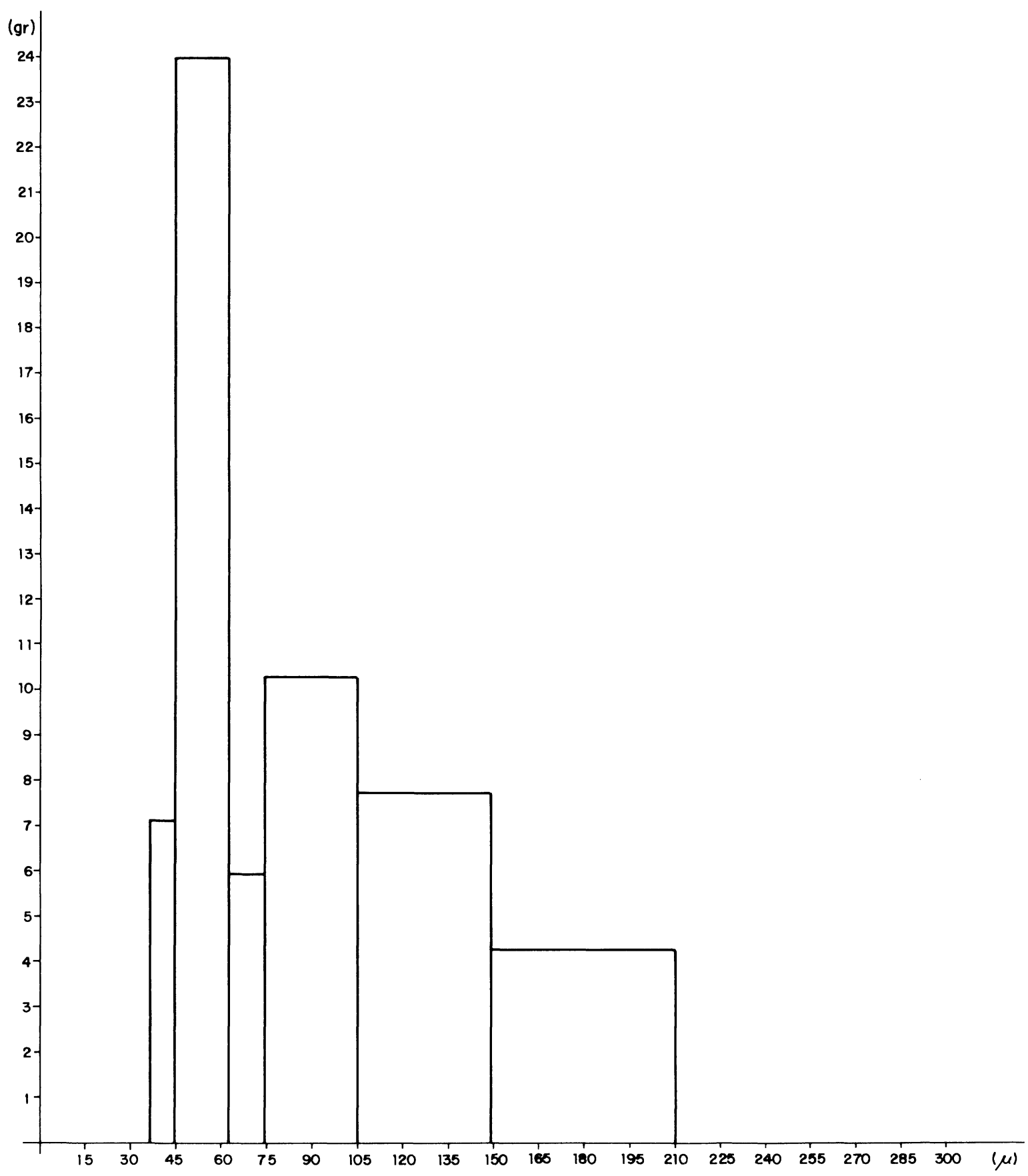

Fig. 2

- Las densidades del sólido (crudo de cemento) y del gas (aire) y la viscosidad del gas son datos que se extraen de cualquier manual "handbook" de física y quimica y que para este caso tienen los siguientes valores: 
$\rho_{\mathrm{g}}=0,0002947 \mathrm{~g} / \mathrm{cm}^{3}$ a $923^{\circ} \mathrm{C}$.

$\rho_{\mathrm{s}}=2,67 \mathrm{~g} / \mathrm{cm}^{3}$.

$\mu=0,0004643 \mathrm{~g} / \mathrm{cm} \cdot \operatorname{seg}$ a $923^{\circ} \mathrm{C}$.

Una vez recopilados todos los datos, no queda más que sustituir en la fórmula dada anteriormente:

$$
\begin{gathered}
{\left[\frac{0,0108 \cdot u_{\mathrm{mf}} \cdot 0,0002947}{0,0004643}\right]^{2}=} \\
=\left[(33,7)^{2}+0,0408 \cdot \frac{(0,0108)^{3} \cdot 0,0002947(2,67-0,0002947) 980}{(0,0004643)}\right]-33,7
\end{gathered}
$$

Despejando:

$$
u_{\mathrm{mf}}=0,39 \mathrm{~cm} / \mathrm{seg}
$$

\section{VELOCIDAD DE ARRASTRE}

La velocidad del fluido que atraviesa un lecho fluidizado está limitada por un lado por la mínima velocidad de fluidización y por otro por la velocidad a la cual el fluido arrastra consigo al sólido. Cuando se llega a la situación en que los sólidos son arrastrados por el fluido, dichos sólidos deben ser reciclados o reemplazados por más material para mantener el equilibrio de la operación.

Este limite superior que se impone a la velocidad del fluido tiene un valor aproximado a la velocidad de caída libre de la partículas y puede calcularse mediante la fórmula:

$$
u_{\mathrm{t}}=\left[\frac{4 g d_{\mathrm{p}}\left(\rho_{\mathrm{s}}-\rho_{\mathrm{g}}\right)}{3 \rho_{\mathrm{g}} C_{\mathrm{d}}}\right]^{1 / 2}
$$

donde $C_{\mathrm{d}}$ es un coeficiente determinado experimentalmente. Existen unas correlaciones entre $C_{\mathrm{d}} \mathrm{y}$ el número de Reynolds que permiten calcular la velocidad terminal o velocidad de arrastre para diferentes intervalos del número de Reynolds:

para $500<\operatorname{Re}<200.000$

$$
u_{\mathrm{t}}=\left[\frac{3,1 g\left(\rho_{\mathrm{s}}-\rho_{\mathrm{g}}\right) d_{\mathrm{d}}}{\rho_{\mathrm{g}}}\right]^{1 / 2}
$$

para $0,4<\operatorname{Rc}<500$

$$
u_{\mathrm{t}}=\left[\frac{\left(\rho_{\mathrm{s}}-\rho_{\mathrm{g}}\right)^{2} g^{2} \cdot 4}{\rho_{\mathrm{g}} \mu \cdot 225}\right]^{1 / 3} \cdot d_{\mathrm{p}}
$$

para $\operatorname{Re}<0,4$

$$
u_{\mathrm{t}}=\frac{g\left(\rho_{\mathrm{s}}-\rho_{\mathrm{g}}\right) d_{\mathrm{p}}^{2}}{18 \mu}
$$


Hay que tener en cuenta que en estas fórmulas, $d_{\mathrm{p}}$ es el tamaño de partícula más pequeño presente en cantidad apreciable.

Pues bien, si se considera la primera opción, es decir $500<\operatorname{Re}<200.000$ entonces:

$$
\begin{gathered}
u_{t}=\left[\frac{3,1 \cdot 980(2,67-0,0002947) \cdot 0,0044}{0,0002947}\right]^{1 / 2} \\
u_{\mathrm{t}}=347,98 \mathrm{~cm} / \mathrm{seg}
\end{gathered}
$$

Se comprueba que efectivamente el número de Reynolds está comprendido entre 500 y 200.0000 .

$$
\operatorname{Re}=\frac{V D \rho}{\mu}=\frac{347,98 \cdot 0,0044 \cdot 2,67}{0.0004643}=8.804
$$

En las otras dos opciones no se cumple que el Re esté comprendido entre aquellos valores para los que se calcula la velocidad de arrastre.

\section{LIMITACIONES DEL LECHO}

La práctica demuestra que en el laboratorio, un lecho funciona bien si se cumplen las siguientes condiciones:

1. La relación longitud-diámetro del lecho debe estar comprendida entre 1 y 3 ; es decir

$$
1 \leqslant L / D \leqslant 3
$$

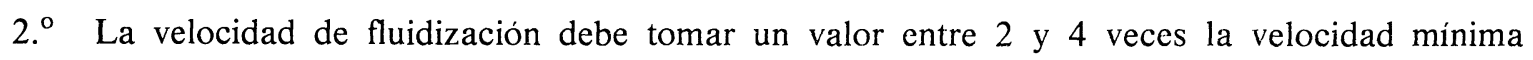
de fluidización:

$$
2 \leqslant u_{\mathrm{f}} / u_{\mathrm{mf}} \leqslant 4
$$

Para el caso que se está estudiando, si se propone una relación $L / D=2,5$ entonces:

$$
V=D^{2 / 4} \cdot L=561,79 \mathrm{~cm}^{3}
$$

ya que $1.500 \mathrm{~g}$ de crudo ocuparán $1.500 / 2,67=561,79 \mathrm{~cm}^{3}$ donde la densidad aparente $(\rho)$ del crudo es $2,67 \mathrm{~g} / \mathrm{cm}^{3}$. Sustituyendo $L=2,5 \mathrm{D}$ en la ecuación del volumen de un cilindro se deduce que:

$$
D=6,6 \mathrm{~cm} \text { y } L=16,42 \mathrm{~cm}
$$

En cuanto a la 2 . $^{\mathrm{a}}$ limitación, si se propone $u_{\mathrm{f}} / u_{\mathrm{mf}}=3$, entonces:

$$
u_{\mathrm{f}}=3 \cdot 0,39=1,17 \mathrm{~cm} / \mathrm{seg}
$$

\section{PERDIDA DE CARGA EN EL LECHO}

La figura $\mathrm{A}$ es un caso típico de un lecho formado por partículas de diferentes tamaños y en donde se representa la pérdida de presión del lecho frente a la velocidad del gas. 


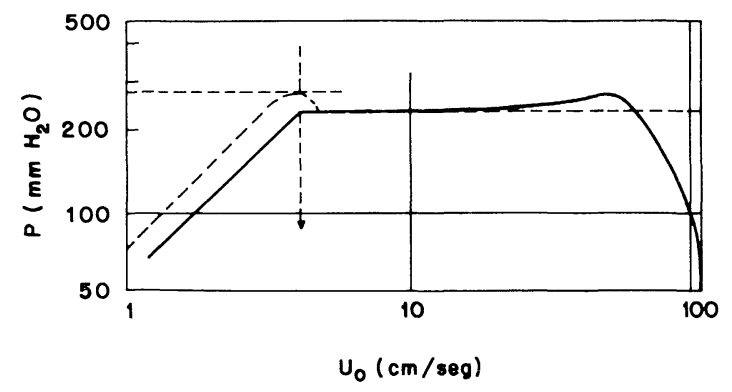

Fig. A

Para velocidades del fluido relativamente bajas, la caída de presión es aproximadamente proporcional a la velocidad del gas alcanzándose normalmente un punto en que $\Delta P$ es máximo. Este valor máximo de $\Delta P$ es ligeramente superior a la presión estática del lecho.

Un posterior aumento de la velocidad del gas tiene como resultado un descenso de la caída de presión estática del lecho, manteniéndose constante este valor de la caída de presión a medida que aumenta la velocidad del gas hasta que comienza el arrastre de las partículas.

La explicación de ese tramo constante de la caída de presión y que corresponde al lecho fluidizado está en el hecho de que el comportamiento hidrodinámico de la fase gas-sólido es comparable al de un líquido: puede deformarse fácilmente sin oponer apreciable resistencia.

Se puede por tanto calcular la pérdida de carga en el lecho según:

$$
(\Delta P)_{\text {lecho }}=\frac{W}{A}=\frac{1.500}{\pi \cdot 3,3^{2}}=43,84 \mathrm{~g} / \mathrm{cm}^{2}
$$

\section{DISTRIBUIDOR}

La experiencia demuestra que la elección de un distribuidor adecuado es de gran importancia para optimizar la calidad de la fluidización. Existen muchos tipos de distribuidores que prestan servicio a diferentes sistemas (operaciones escala laboratorio, operaciones escala industrial, cargas pesadas, etc.).

También demuestra la experiencia, que la caída de presión a través del distribuidor debe ser considerablemente mayor que la resistencia al reordenamiento del gas entrante. Se recomienda que la caída de presión en el distribuidor se aproxime al $10 \%$ de la caída de presión a través del lecho; es decir

$$
\frac{(\Delta P)_{\text {dist. }}}{(\Delta P)_{\text {lecho }}} \approx 0,10
$$

Sin embargo en el lecho objeto del diseño se partirá de una relación $(\Delta P)_{\text {dist. }} /(\Delta P)_{\text {lecho }}=1$ ya que al tratarse de un lecho fluidizado para laboratorio no importa tanto el ahorro energético como la calidad de la fluidización. Así pues:

$$
(\Delta P)_{\text {dist. }}=(\Delta P)_{\text {lecho }}=43,84 \mathrm{~g} / \mathrm{cm}^{2}
$$

Con esto, con los datos anteriormente deducidos y considerados la velocidad de entrada del gas por los orificios del distribuidor $\left(u_{\mathrm{or}}\right)$ igual a la velocidad real de fluidización, es decir:

$$
u_{0}=u_{\mathrm{f}}=1,17 \mathrm{~cm} / \mathrm{seg}
$$

se calcula el Reynolds del fluido

$$
\operatorname{Re}=\frac{D \rho_{\mathrm{g}} u_{0}}{\mu}=\frac{6,6 \cdot 0,0002947 \cdot 1,17}{0,0004643}=4,90
$$


De la figura $B$ en la que se representa el-coeficiente de orificio frente al número de Reynolds se deduce dicho coeficiente para el caso que se está tratando:

para: $\quad \operatorname{Re}=4,90 \quad C_{\mathrm{d}}^{\prime}=0,35$

La velocidad del gas a través de los orificios viene determinada por:

$$
u_{\mathrm{or}}=C_{d}^{\prime}\left(\frac{2 g_{\mathrm{c}}(\Delta P)_{\mathrm{dist.}}}{\rho_{g}}\right)^{1 / 2}
$$

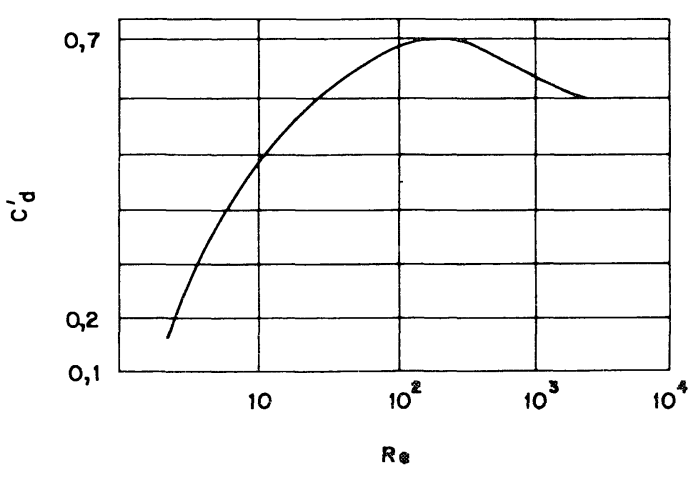

Fig. B

Sustituyendo:

$$
u_{\text {or }}=0,35\left[\frac{2 \cdot 980 \cdot 43,84}{0,0002947}\right]^{1 / 2}=5.976,42 \mathrm{~cm} / \mathrm{seg}
$$

Se puede por tanto deducir la fracción de área abierta:

$$
u_{\mathrm{o}} / u_{\text {or }}=1,17 / 5.976,42=0,000195<>0,0195 \%
$$

La relación entre el número y el tamaño de los orificios viene dada por:

$$
u_{\mathrm{o}}=\pi / 4 d_{\mathrm{or}}^{2} u_{\mathrm{or}} N_{\mathrm{or}}
$$

De este modo, para diversos tamaños de orificios encontramos:

\begin{tabular}{|l|lllll|}
\hline$d_{\text {or }}(\mathrm{cm})$ & 0,01 & 0,02 & 0,05 & 0,1 & 0,3 \\
\hline$N_{\text {or }}\left(\mathrm{n} . \% / \mathrm{cm}^{2}\right)$ & 2,48 & 0,62 & 0,1 & 0,02 & 0,0027 \\
\hline
\end{tabular}

Orificios demasiado pequeños son susceptibles de taponarse, mientras que orificios demasiado grandes pueden dar lugar a distribuciones desiguales del gas.

En este caso y por estas razones se escogerá un diámetro de orificio de $0,01 \mathrm{~cm}$.

\section{TRANSMISION DE CALOR EN EL LECHO}

En la industria, el mantener durante una operación determinada unas condiciones de temperatura prefijadas, constituye un problema de importancia capital.

En toda transformación química se desprende o absorbe calor. El rendimiento y la velocidad de una reacción química están determinados por la temperatura.

Unas veces es necesario calentar, otras enfriar, es decir siempre transmitir calor en uno $u$ otro sentido.

Muchas reacciones que se desarrollan a temperatura muy alta o muy baja, sólo pueden realizarse económicamente si se recupera el calor o frío acumulados en los productos de reacción y si se evitan las pérdidas durante las mismas, o sea que de nuevo surgen problemas de transmisión de calor. 
También en muchos procesos físicos se presentan problemas de transmisión de calor. Así por ejemplo: Diseño y funcionamiento de generadores de vapor, hornos, precalentadores, etc. en numerosas y variadas industrias.

En ocasiones, el objetivo principal es alcanzar el flujo máximo y económico de calor. Otras veces se pretende economizar calor como es el caso en los intercambiadores, recuperadores y regeneradores de calor.

Hay ocasiones que interesa minimizar las pérdidas de calor mediante aislantes térmicos.

Con frecuencia resulta importante alcanzar todos estos objetivos conjuntamente; es pues evidente que las leyes que regulan la transmisión de calor a través de los cuerpos y de unos a otros resultan de la máxima importancia.

Por las características específicas de este proyecto, el problema de la transmisión de calor en el interior del lecho fluidizado se tratará de un modo sencillo. Sólo se tendrá en cuenta el intercambio de calor entre el gas (aire) y el lecho y no el intercambio entre el gas y las paredes del tubo donde se desarrolla la operación.

Puesto que se trata de una operación a escala de laboratorio, no será prioritario obtener un gran rendimiento económico en materia energética.

\section{CALCULOS}

Primeramente, se planteará un sencillo balance de calor:

$$
\left[\begin{array}{l}
\text { Calor absorbido por } \\
\text { el sólido hasta al- } \\
\text { canzar la temperatu- } \\
\text { ra deseada }
\end{array}\right]+\left[\begin{array}{l}
\text { Calor necesario pa- } \\
\text { ra lograr la descom- } \\
\text { posición del } \mathrm{CaCO}_{3}
\end{array}\right]=\left[\begin{array}{l}
\text { Calor cedido por el } \\
\text { gas }
\end{array}\right]
$$

dicho en otros términos:

$$
m_{\mathrm{s}} C_{\mathrm{s}}\left(T_{\mathrm{es}}-T_{\mathrm{ss}}\right)+m_{\mathrm{a}}(-\Delta h)=-m_{\mathrm{g}} C_{\mathrm{g}}\left(T_{\mathrm{cg}}-T_{\mathrm{sg}}\right)
$$

o bien

$$
m_{\mathrm{s}} C_{\mathrm{s}}\left(T_{\mathrm{ss}}-T_{\mathrm{es}}\right)+m_{\mathrm{a}}(\Delta H)=m_{\mathrm{g}} C_{\mathrm{g}}\left(T_{\mathrm{eg}}-T_{\mathrm{sg}}\right)
$$

en donde

$m_{\mathrm{s}}=$ masa del sólido;

$C_{\mathrm{s}}=$ calor específico del sólido;

$\mathrm{T}_{\mathrm{es}}=$ temperatura inicial del sólido;

$T_{\text {ss }}=$ temperatura final del sólido;

$m_{\mathrm{a}}=$ masa de sólido que se descompone;

$\Delta H=$ entalpía de descomposición del sólido;

$m_{\mathrm{g}}=$ masa del gas;

$C_{\mathrm{g}}=$ calor específico del gas;

$T_{\text {eg }}=$ temperatura de entrada del gas;

$T_{\mathrm{sg}}=$ temperatura de salida del gas. 
En el balance entálpico se ha tenido en cuenta la descomposición térmica del carbonato cálcico

$$
\mathrm{CaCO}_{3} \stackrel{\mathrm{T}}{\longrightarrow} \mathrm{CaO}+\mathrm{CO}_{2}
$$

Porque aproximadamente el $75 \%$ de la masa de sólido que hay en el lecho sufrirá esa descomposición que sólo se realiza con un elevado aporte de energía.

El término $m_{\mathrm{a}}(-\mathrm{A} H)$ tiene en cuenta este hecho.

Para resolver ese balance entálpico se hace lo siguiente:

hay unos datos que vienen fijados por el propio sistema tales como:

$$
C_{\mathrm{s}}=0,2 \mathrm{cal} / \mathrm{g} \quad C_{\mathrm{g}}=0,257 \mathrm{cal} / \mathrm{g} \quad \Delta H=-178 \mathrm{KJ} / \mathrm{mol} \quad \text { y } \quad m_{\mathrm{a}}=0,75 m_{\mathrm{s}}
$$

El resto de los datos son definidos dependiendo de las caracteristicas del sistema que se desea diseñar y teniendo en cuenta que posteriormente con esos datos habrán de verificarse las ecuaciones de transmisión de calor.

En resumen: habrá que hacer un cálculo iterativo hasta que se cumplan simultáneamente el balance entálpico y las leyes de la transmisión de calor y que estas a su vez satisfagan las condiciones de fluidización.

Así pues se fijan las temperaturas iniciales del sólido y del gas en 750 y $1.100^{\circ} \mathrm{C}$ respectivamente y las temperaturas finales en 950 y $975^{\circ} \mathrm{C}$. Se fija también una masa de sólido de $1,5 \mathrm{Kg}$.

Sustituyendo los datos en el balance anteriormente planteado se tiene que:

$$
\begin{gathered}
1.500(\mathrm{~g}) \cdot 0,2(\mathrm{cal} / \mathrm{g}) \cdot(950-750)+11,25(\text { moles }) \cdot 42.532,8(\mathrm{cal} / \mathrm{mol})= \\
\left.=m_{\mathrm{g}}(\mathrm{g}) \cdot 0,257(\mathrm{cal} / \mathrm{g}) \cdot 1.100-975\right)
\end{gathered}
$$

de donde se obtiene:

$$
m_{\mathrm{g}}=16.762,4 \mathrm{~g}
$$

Como se verá, el término $m_{\mathrm{a}}(-\Delta H)$ se ha puesto en las unidades convenientes:

$$
\begin{gathered}
m_{\mathrm{a}}=0,75 \cdot m_{\mathrm{s}}=0,75 \cdot 1.500=1.125 \mathrm{~g} \\
1.125 \mathrm{~g} \text { de } \mathrm{CaCO}_{3}<>11,25 \text { moles de } \mathrm{CaCO}_{3} \\
178 \mathrm{KJ} / \mathrm{mol}: 4,185=42,5328 \mathrm{Kcal} / \mathrm{mol}
\end{gathered}
$$

La masa de aire $\left(m_{\mathrm{g}}\right)$ calculada, necesaria para calentar el sólido desde 750 hasta $950^{\circ} \mathrm{C}$ ocupa un volumen dado por:

$$
V=\frac{m_{\mathrm{g}}}{\rho_{\mathrm{g}}}=\frac{16.762,4(\mathrm{~g})}{0,0002947\left(\mathrm{~g} / \mathrm{cm}^{3}\right)}=56.879 .538,5 \mathrm{~cm}^{3}<>56,879 \mathrm{~m}^{3}
$$

Si se fija un tiempo de fluidización de 1 hora, con el que se tiene la certeza de que se cumplirá el balance entálpico propuesto, ese volumen de aire será equivalente a pasar a través del lecho un caudal de aire a $1.100^{\circ} \mathrm{C}$ de $15,8 \mathrm{l} / \mathrm{seg}$. 
El calor, por tanto, necesario para aumentar la temperatura del sólido desde $750^{\circ} \mathrm{C}$ hasta $950^{\circ} \mathrm{C}$ es:

$$
Q=1.500 \cdot 0,2 \cdot 200+11,25 \cdot 42.532,8=538.494 \mathrm{cal}
$$

Se comprobará a continuación por medio de las ecuaciones de transmisión de calor que efectivamente ese número de calorías es el necesario para que ese sólido aumente su temperatura desde 750 hasta $950^{\circ} \mathrm{C}$.

La cantidad de calor puesta en juego en la operación objeto de estudio viene dada por la expresión:

$$
Q=A h_{\mathrm{p}} \Delta T
$$

en donde:

$\mathrm{A}=$ área de intercambio de calor;

$h_{\mathrm{p}}=$ coeficiente de transmisión de calor;

$\Delta T=$ incremento de temperatura.

\section{Cálculo del área de intercambio de calor}

Se supondrá que el único intercambio de calor existente será aquel que se produzca entre el fluido y el sólido a calentar, no interviniendo las paredes del recipiente que los contiene.

De este modo, ya que la masa de sólido es conocida $(1.500 \mathrm{~g})$ y también lo es su densidad $\left(2,67 \mathrm{~g} / \mathrm{cm}^{3}\right)$, el volumen que ocupa el sólido será conocido

$$
\left(V=1.500 / 2,67=561,79 \mathrm{~cm}^{3}\right)
$$

También se conoce el tamaño medio de las partículas $(r=0,0054 \mathrm{~cm})$.

Con estos datos podemos calcular el número de partículas de sólido:

$$
\begin{gathered}
V=n \cdot 4 / 3 \pi r^{3} ; \quad n=\frac{3 V}{4 \pi r^{3}} \\
n=\frac{3 \cdot 561,79}{4 \cdot \pi \cdot(0,0054)^{3}}=851.734 .258 \text { partículas }
\end{gathered}
$$

La superficie de intercambio de calor vendrá dada por:

$$
A=n \cdot 4 \pi r^{2}
$$

entonces

$$
\begin{gathered}
A=851.734 .258 \cdot 4 \cdot \pi \cdot(0,0054)^{2} \\
A=312.105,5 \mathrm{~cm}^{2}
\end{gathered}
$$

Conocida el área de intercambio de calor, sólo resta por conocer el coeficiente de transmisión de calor: 


\section{Cálculo del coeficiente de transmisión de calor}

El coeficiente de transmisión de calor $h_{\mathrm{p}}$ entre la superficie de una esfera de diámetro $d_{\mathrm{p}} \mathrm{y}$ un fluido a través del cual se está moviendo con una velocidad relativa $N_{0}$ viene dado por:

$$
\mathrm{Nu}=\frac{h_{\mathrm{p}} d_{\mathrm{p}}}{K}=2+0,6 \operatorname{Pr}^{1 / 3} \operatorname{Re}^{1 / 2}
$$

donde:

$\mathrm{Nu}=$ número de Nusselt;

$\operatorname{Pr}=$ número de Prandtl;

$\mathrm{Re}=$ número de Reynolds;

$h_{\mathrm{p}}=$ coeficiente de transferencia de calor;

$d_{\mathrm{p}}=$ diámetro de partícula;

$K=$ conductividad térmica del fluido.

Por otra parte:

$$
\operatorname{Pr}=\frac{\mu \mathrm{C}_{\mathrm{p}}}{K} \quad \mathrm{y} \quad \mathrm{Re}=\frac{V D \rho}{\mu}
$$

donde $\mu, C_{\mathrm{p}}, \rho$ y $V$ son respectivamente la viscosidad, el calor específico, la densidad y la velocidad del gas.

Se puede considerar éste, el caso de una serie de partículas esféricas moviéndose en el seno de un gas que atraviesa el lecho. Asi pues:

$$
h_{\mathrm{p}}=\frac{\left(2+0,6 \operatorname{Pr}^{1 / 3} \operatorname{Re}^{1 / 2}\right) \cdot K}{d_{\mathrm{p}}}
$$

El número de Reynolds que ya fue calculado anteriormente vale 4,90.

En cuanto al número de Prandtl:

$$
\operatorname{Pr}=\frac{\mu C_{\mathrm{p}}}{K}=\frac{0,0004643(\mathrm{~g} / \mathrm{cm} \cdot \mathrm{seg}) \cdot 0,257(\mathrm{cal} / \mathrm{g})}{0,0000568(\mathrm{cal} / \mathrm{seg} \cdot \mathrm{cm})}=2,1
$$

Por tanto:

$$
\begin{gathered}
h_{\mathrm{p}}=\frac{\left.\left[2+0,6(2,1)^{1 / 3}\right] \cdot(4,9)^{1 / 2} \cdot 0,0000568 \mathrm{cal} / \mathrm{seg} \cdot \mathrm{cm}\right)}{0.0108(\mathrm{~cm})} \\
h_{\mathrm{p}}=0,0083 \mathrm{cal} / \mathrm{seg} \cdot \mathrm{cm}^{2}
\end{gathered}
$$

\section{Cálculo del número de calorias}

Volviendo a la ecuación que da la cantidad de calor en función del área y del coeficiente de transmisión de calor se tiene que:

$$
Q=A h_{\mathrm{p}} \mathrm{A} T
$$




$$
\begin{aligned}
& Q=312.105,5 \cdot 0,0083 \cdot 200 \\
& Q=518.095 \mathrm{cal}
\end{aligned}
$$

Comparando este número de calorías con las 538.494 deducidas en el balance entálpico se observa una buena aproximación que puede dar por válidas las variables de diseño fijadas a las que se han llegado como ya se indicó anteriormente por medio de un cálculo iterativo.

\section{BIBLIOGRAFIA CONSULTADA}

- Dipl. Ing. WALTER H. DUDA: Manual Tecnológico del Cemento. Editores Técnicos Asociados, S. A. (1977).

- J. H. PERRY: Chemical Engineers' Handbook, third ed., McGraw-Hill Book Company, New York (1963).

- G. G. BROWN et al: Unit Operations, John Wiley and Sons, New York (1963).

- M. LEVA: Fluidization, McGraw-Hill Book Company, New York (1959).

- V. VANECEK, M. MARKVART and R. DRBOHLAV: Fluidized Bed Drying. Translated by J. Landau, Leonard Hill, London (1966).

- D. KUNII and O. LEVENSPIEL: Ind. Eng. Chem., Process Design and Development, 7 (Oct. 1968).

-- S. S. ZABRODSKY: Hidrodinamics and Heat Transfer in Fluidized Beds, Translated by F. A. Zenz, M.I.T. Press, Cambridge, Mass (1966).

\section{publicaciones del i.e.t.c.c.}

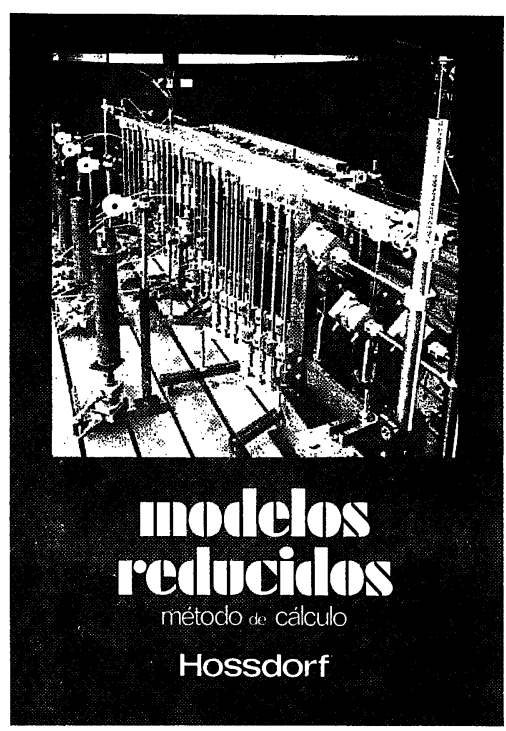

Modelos reducidos. Método de cálculo

H. Hossdorf, Ingeniero Civil

La técnica de los ensayos en modelos reducidos de estructuras sufre hoy dia una decisiva metamorfosis. Hasta hace poco era un medio más bien de artesania, que no siempre era tomado en serio por los academicos teorizantes Dara comprender el comportaque se acudió las más estructuras complejas y a ultimo remedio debido a las indiscutibles insuficien ultimo remedio debido a sus indiscutibles insuficien cias. Sin embargo, en poco tiempo y gracias a su conexión con los ordenadores digitales, se ha transque no puede quedar a un lado en la váctica diaria del Ingeniero Proyectista.

Un volumen encuadernado en cartoné plastificado con lomo de tela, de $17 \times 24 \mathrm{~cm}$, compuesto de 250 páginas, 158 figuras y fotografias.

Precios: 1.800 ptas.; \$ USA 26.00.

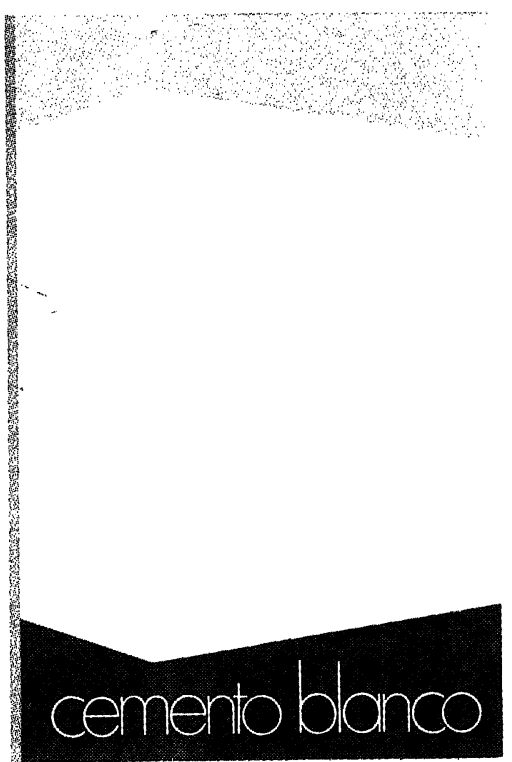

Cemento blanco

Julián Rezola

Ingeniero Quimico Dipl. I. Q. S.

Sabido es que existe una extensa y documentada bibliografia sobre el cemento gris: en cambio, no puede decirse lo mismo acerca del cemento portland blanco, ya que los escritos existentes se refieren tan de aquél.

El autor nos ofrece sus profundos conocimientos y su larga experiencia tanto en laboratorio como en fabricación.

La parte descriptiva del libro se complementa con gráficos, diagramas y fotografias de gran utilidad, gráficos, diagramas y fotografias de gran utilidad, este aglomerante.

Un volumen encuadernado en cartoné policerado, de $17,4 \times 24,3 \mathrm{~cm}$, compuesto de 395 páginas. numerosas figuras, tablas y ábacos.

Precios: España, 1.700 ptas.; extranjero, $\$ 34$

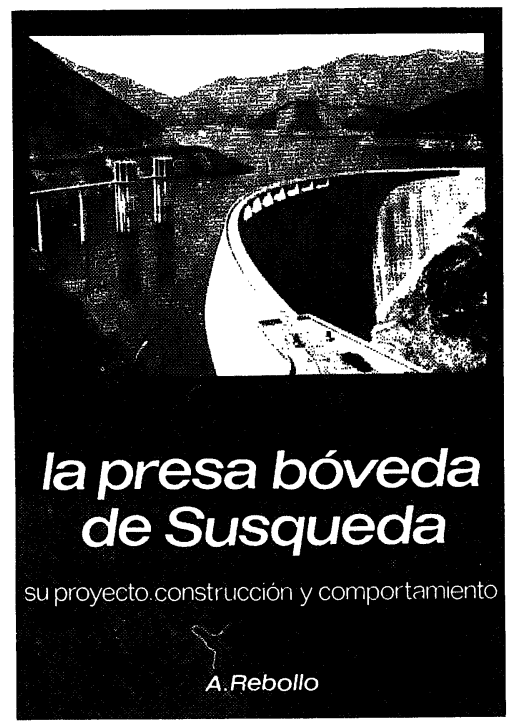

La presa bóveda de Susqueda

A. Rebollo,

Dr. Ingeniero de Caminos

El esfuerzo del constructor de presas se sitúa por su pretensión de perennidad, a contracorriente de las tendencias de la civilización actual, caracte rizada por lo fungible. Pueden evocarse las 10.000 grandes. presas en funcionamiento o en construcción que están envejeciendo y reclaman los cuidados gerontológicos para mantener y perfeccionar su servicio y garantizar su inalienable pretensión de perennidad. En la medida en que todas nuevas obras, grandes o pequeñas, son portadoras de riesyos ecológicos $y$, a veces, catastróticos, que aumentan con el envejecimiento, la gerontologia de las presas es todo un emplazo. La accion adelantada de Arturo Rebollo en este terreno marca un camino a seguir para todos los que aman su propia obra con Un volumen encuadernado en cartonè plastificado 408 páginas, 330 figuras y fotografias y 39 tablas. Precios: 1.700 ptas.; extranjero, \$ USA 24.00. 